\title{
LIMITED STREAMER TUBES FOR THE BABAR INSTRUMENTED FLUX RETURN UPGRADE
}

\author{
CHANGGUO LU \\ ON BEHALF OF THE BABAR LST GROUP \\ Princeton University \\ Princeton, NJ 08544, USA
}

\begin{abstract}
Starting from the very beginning of their operation the efficiency of the RPC chambers in the BaBar Instrumented Flux Return (IFR) has suffered serious degradation. After intensive investigation, various remediation efforts had been carried out, but without success. As a result the BaBar collaboration decided to replace the dying barrel RPC chambers about two years ago. To study the feasibility of using the Limited Streamer Tube (LST) as the replacement of RPC we carried out an R\&D program that has resulted in BaBar's deciding to replace the barrel RPC's with LST's. In this report we summarize the major detector R\&D results, and leave other issues of the IFR system upgrade to the future publications.
\end{abstract}

\section{Status of the BaBar IFR system}

The efficiency of the original Resistive Plate Chambers (RPC's) in the BaBar Instrumented Flux Return (IFR) has seriously degraded, now averaging 40\% (see figure 1). After intensive investigation, various remediation efforts had been carried out, but without success [1]. The BaBar collaboration decided to replace the dying barrel RPC chambers about two years ago, and during the summer of 2002 the forward endcap IFR system was upgraded. To increase absorber thickness, we replaced 5 layers of the

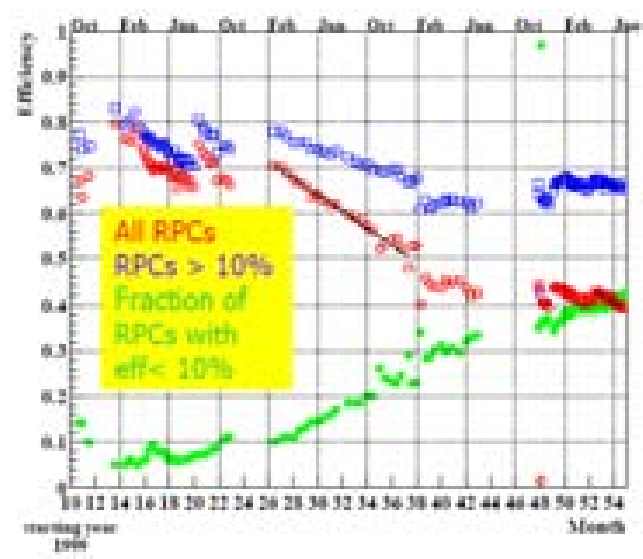

Figure 1. Efficiency degradation of BaBar RPCs in October 1999 to June 2003. original RPCs with 2-cm-thick brass plates; and new RPCs have replaced the sick RPCs for the other layers. However, the situation in the barrel IFR continued to deteriorate to the point that it provides inadequate muon identification for some applications, and will become a serious problem in the future. Starting from the summer of 2004, the upgrade of BaBar barrel IFR system has begun. Six brass layers will replace the RPCs to increase the ID efficiency,

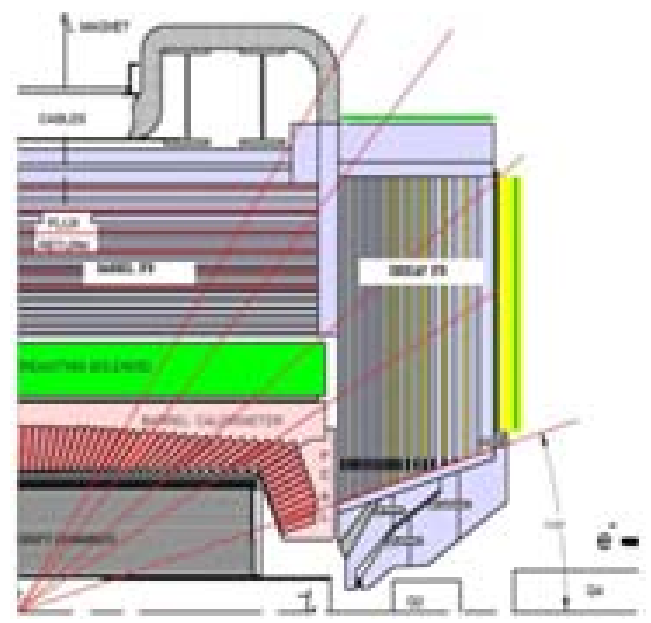

Figure 2. BaBar endcap and barrel IFR system.

the remaining 12 RPC layers will be replaced by LSTs. In 2004 two of the six sectors of the 
barrel IFR were upgraded; the remaining four sectors will be done in summer 2005. Figure 2 shows the upgraded BaBar endcap and barrel IFR systems. The red lines in the barrel IFR indicate locations of the new brass layers .

\section{Why does BaBar choose LST to replace RPC?}

The simplicity and low cost of RPC technology have resulted in more and more experiments choosing them over LST for relevant applications. However, puzzling features of RPC behavior are still not understood. New and unresolved problems continue to emerge. Completely understanding the physics and chemistry of RPC operation was not in the scope of urgent BaBar decision making. On the other hand, LSTs are conceptually well understood and have been used in more than a dozen HEP experiments with generally good results [2-10]. Our own successful prototype tests have demonstrated robustness against the highest expected rates, and the integrated charge over the experimental lifetime. All of these led BaBar to choose LST for the barrel IFR upgrade.

\section{Choice of the tube geometry}

Due to the limited gap space ( 22mm) available in the BaBar barrel IFR, to reduce the intrinsic effect of LST geometrical dead space on its efficiency, we have to increase the thickness of the gas gap as much as possible. There were two options:

single layer of large cells $-17 \times 15 \mathrm{~mm}^{2}$;

double layer of small cells $-9 \times 8 \mathrm{~mm}^{2}$. (There is insufficient space for a double layer of $9 \times 9 \mathrm{~mm}^{2}$ "standard” cell LST's and still allow room for tube walls and readout planes.) We performed electric field calculations comparing two geometries for their e-field stability in two situations: anode wire off center by $0.5 \mathrm{~mm}$ towards the bottom; anode wire diameter increased by $1 \%$. The calculation results are summarized in the table 1.
Based on this comparison the conclusions are: The effect of anode wire diameter variation is comparable, but the large cell is not as sensitive as small cell to the anode wire displacement. Other important advantages of the large cell design are fewer cells (only 30\% of small cell design), and much better overall mechanical rigidity. These considerations led us to choose the large cell geometry.

Table 1. E-field stability for two geometries.

\begin{tabular}{|c|c|c|c|c|}
\hline & $\begin{array}{l}\text { Small cell LST } \\
9 \times 8 \mathrm{~mm}\end{array}$ & $\begin{array}{l}\text { Large cell } \\
\text { LST } \\
17 \text { x } 15 \text { mm } \\
\end{array}$ \\
\hline \multicolumn{3}{|c|}{ Anode voltage(V) } & 4700 & 5500 \\
\hline \multirow{2}{*}{\multicolumn{2}{|c|}{$\begin{array}{l}\text { Anode wire off } \\
\text { center by } 0.5 \mathrm{~mm} \\
\text { towards bottom } \\
\text { side }\end{array}$}} & $\mathrm{E} / \mathrm{E}$ & $1.89 \%$ & $0.876 \%$ \\
\hline & & V & $88.8 \mathrm{~V}$ & $48 \mathrm{~V}$ \\
\hline \begin{tabular}{|l|} 
Anode wire \\
diameter \\
increases \\
$1 \%$ of \\
$100 \mathrm{~m}$ \\
\end{tabular} & \multicolumn{2}{|c|}{\begin{tabular}{|l}
$\mathrm{V} / \mathrm{V}$ \\
(Effective \\
relative \\
voltage \\
change ) \\
\end{tabular}} & $-0.79 \%$ & $-0.81 \%$ \\
\hline
\end{tabular}

\section{Aging test on LST's with two different cathode coatings.}

There are two types of cathode coating used in making LST tubes:

A. MIBK (Methyl-Isobutyl-Ketone) based graphite paint

We have tested an LST made by the U. Houston Scarf facility: standard LST size $9 x 9 \mathrm{~mm}^{2}$ with MIBK based graphite paint. A charge dose > $0.75 \mathrm{C} / \mathrm{cm}^{2}$ has been accumulated. Up to this limit no significant degradation of operation has occurred (no significant gain loss; no selfsustaining discharge).

B. Water based paint

For the BaBar upgrade we are using LSTs made by PolHiTech* ${ }^{*}$ They are painted with water based graphite paint. So far the total accumulated charge dose is $\sim 0.6 \mathrm{C} / \mathrm{cm}$. The current, and hence the gas gain remains stable. The maximum charge dose expected by 2010

\footnotetext{
* An Italian company at S. P. Turanense Km 44,400, 67061 Carsoli(AQ), Italy
} 
for the barrel LST's is $0.12 \mathrm{C} / \mathrm{cm}$, so we have a quite large safety margin.

\section{Characteristics of two types of graphite paints}

MIBK based graphite paint - Used in Majority of previous experiments:

- Good adhesion,

- Stable resistivity, not sensitive to humidity,

- But hazardous.

Water based graphite paint:

Not hazardous -- greatly simplifies the manufacturing facility,

Weak adhesion of the paint on the PVC substrate. It is essential to clean the PVC profile carefully to achieve reliable, strong adhesion of the coating skin.

Resistivity of the coating depends greatly upon humidity.

Resistivities of water-based and MIBK-based paint have very different sensitivity to the ambient humidity. Our test shows for the relative resistivity increase vs. the relative humidity the water based paint is 20 times larger than MIBK based paint.

\section{Source scan test}

How to find the internal defects quickly without opening the tube? Following Opal's experience [11], we scan each cell with a radioactive source as shown in figure 3 , subjecting it to local currents far in excess of normal operation. This has proved to be a powerful tool: for good tubes, the current remains steady while the source is present, and returns to zero as soon as the source is removed. For regions of bad surface, however, the source might trigger a large, self-sustaining current.

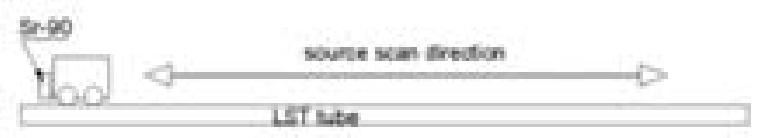
weakness. In figure 4 we show the effect of a pinnacle and a bald spot on the surface E-field

\section{Common failure modes}

LST's are not usually manufactured in a clean room because of cost, resulting in less-thanperfectly clean interior surfaces that can cause various problems during LST commissioning. To clean up the interior of the LST, HV conditioning is one of the most important procedures: gradually raise $\mathrm{HV}$ in steps until it reaches the maximum value without drawing abnormal current. During that period the current may be up and down several times, but in most cases it will relax to a low value after sufficient conditioning.

The most common failure mode encountered in LST Q/C is the self-sustaining discharge.

We have found at least three defects can cause self-sustaining discharge:

- "pinnacles" on the graphite surface;

- bald spots on the graphite surface;

- $\quad$ dirty anode wire surface.

We have tested the behavior of a pinnacle and a bald spot ( $\sim 0.35 \mathrm{~mm}$ in dimension) under a $\mathrm{Sr}-$ 90 source irradiation. The total current of the irradiated spot is $\sim 2 \mu \mathrm{A}$. Upon opening of the source shutter it only takes a few seconds for the current to jump to self-sustaining mode, and the high current persists even after the source is removed. For a good surface, the current remains stable throughout more than 40 minutes of irradiation, and drops to the background value immediately the source is shut off. This clearly indicates that good surfaces can handle very high rates.

Why can pinnacles and bald spots cause selfsustaining discharge? Electrostatic calculations show that at the edge of a bald spot or tip of a pinnacle the electric field strength is much higher than normal, and hence field emission would be easier at these areas. Under normal low-radiation operation the positive feedback is insufficient to trigger the self-sustaining discharge, but the intensive source radiation can reveal this

Figure 3. Source scan test setup. 
distribution (the dimensions of these defects are $\sim 1 \mathrm{~mm}$ ).

In figure 5 we show a microscope image of a typical pinnacle.

\section{Remediation of hot spots due to dirty wire surfaces.}

Often when one opens an LST with bad plateau no obvious defect can be found. In this case the hot spot is most likely due to dirty wire surface. We can find hot spots with a pick-up coil placed on the top of the tube.

Cleaning the wire surface with ethanol/acetone can remove this type of hot spot, but it is laborious, somewhat risky, and impossible once the LST's have been installed into BaBar. We have found an alternative way to cure this problem without opening the tube: negative HV treatment. When we reverse the polarity of the $\mathrm{HV}$ and gradually increase the voltage, there are large current fluctuations for a while even when we increase the voltage by a small step. But eventually the current calms down in most cases. Our experience shows if we can raise the $-\mathrm{HV}$ up to $-4100 \mathrm{~V}$ without drawing large current (after the initial fluctuations), in most cases the hot spot has been cured, and much better plateaus can be obtained [12].

\section{Conclusions}

Many experiments have successfully used LST technology for large scale muon detection and hadron calorimeters;

A notorious problem for LST is setting up an adequate $\mathrm{Q} / \mathrm{C}$ procedure. To be successful in this regard our R\&D has provided various valuable insights, which provided better understanding of the LST technology;

- We have explored the most common failure mode: self-sustaining discharge, and have studied the role of graphite coating surface pinnacles, bald spots and dirty wire surfaces in the discharge;

- We have developed an effective negative corona discharge method to cure the shortplateau LST.

\section{Acknowledgments}

This work was supported by Istituto Nazionale di Fisica Nucleare (INFN) and US Department of Energy (DoE).

\section{References}

1. F. Anulli et al., NIM A508(2003) 128

2. E. Iarocci et al. NIM 217(1983) 30

3. G. Bauer et al., NIM A253(1987) 179

4. C. Bari et al., NIM A264(1988) 5

5. K. Lau et al., Symposium on detector research and development for the SSC Oct. 15-18, 1990, Fort Worth, Texas, pp. 640-642

6. The ALEPH Collaboration, NIM A294(1990) 121

7. A.C. Benvenuti et al., NIM A290(1990) 353

8. OPAL collaboration, NIM A305(1991) 275

9. G. Abbiendi et al., NIM A333(1993) 342

10. H Akikawa et al., NIM A499(2003) 537

11. F. Fabbri et al., CERN/EP 87-134

12. Negative HV treatment was used in constructing the BaBar drift chamber, and by several other groups.
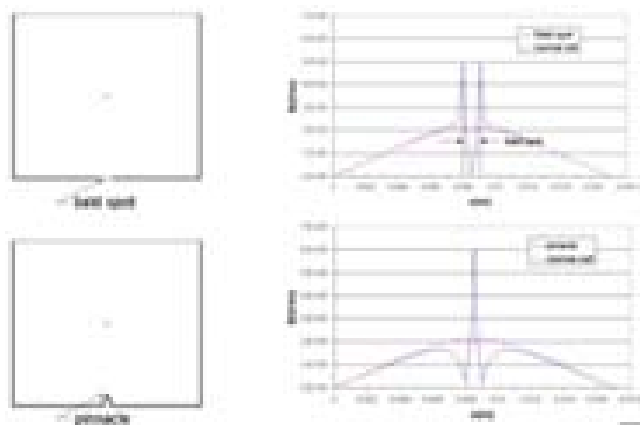

Figure 4. E-field calculation for pinnacle and bald spot The dimension of pinnacle and bald spot is $1 \mathrm{~mm}$.

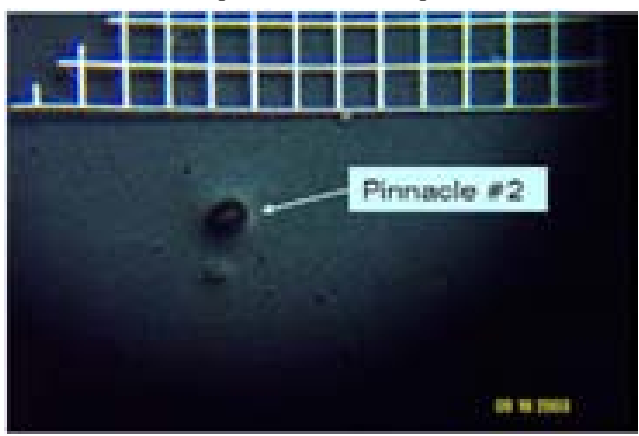

Figure 5. Image of a pinnacle under a microscope. The pitch of the mesh in the picture is $0.35 \mathrm{~mm}$. 